\title{
Pulmonary resection after immunotherapy
}

\author{
Prabhu Sasankan ${ }^{1}$, Jonathan Hyde ${ }^{1}$, Simeng Wang ${ }^{2}$, Travis C. Geraci ${ }^{3}$, Robert J. Cerfolio ${ }^{3}$ \\ ${ }^{1}$ New York University School of Medicine, NYU Langone Health, New York, NY, USA; ${ }^{2}$ Department of General Surgery, ${ }^{3}$ Department of \\ Cardiothoracic Surgery, New York University Langone Health, New York, NY, USA \\ Contributions: (I) Conception and design: TCGeraci, RJ Cerfolio; (II) Administrative support: RJ Cerfolio; (III) Provision of study materials or \\ patients: All authors; (IV) Collection and assembly of data: RJ Cerfolio; (V) Data analysis and interpretation: All authors; (VI) Manuscript writing: All \\ authors; (VII) Final approval of manuscript: All authors. \\ Correspondence to: Travis C. Geraci, MD. Department of Cardiothoracic Surgery, New York University Langone Health, 550 1st Avenue, 15 th Floor, \\ New York, NY 10016, USA. Email: travis.geraci@nyulangone.org.
}

\begin{abstract}
The efficacy of immunotherapy for patients with late stage non-small cell lung cancer (NSCLC) has inspired its application for earlier stage disease. Immunotherapy as a neoadjuvant arm of a multimodality treatment strategy has shown promise in recent prospective trials. Pulmonary resection after immunotherapy can be challenging due to the development of mediastinal fibrosis and/or pneumonitis. Recent trials have established the safety and feasibility of minimally invasive pulmonary resection for patients after immunotherapy. The toxicity of immunotherapies creates new challenges for both oncologists and thoracic surgeons. For patients undergoing a minimally invasive operation, the conversion rate to an open thoracotomy remains limited. In this review, we will summarize the current data for neoadjuvant immunotherapy and discuss early experiences with pulmonary resection after immunotherapy. We also include an example video or a robotic left upper lobe lobectomy after immunotherapy with reconstruction and reinforcement of the left upper lobe bronchus. With future studies ongoing, we anticipate a growing cohort of patients presenting for surgical resection after immunotherapy. Questions remain unanswered in patients who receive neoadjuvant therapy, including the optimal timing for surgery after therapy, whether immunotherapy is given alone or in combination with traditional cytotoxic chemotherapy, or whether patients with a complete pathologic response require surgical resection.
\end{abstract}

Keywords: Immunotherapy; neoadjuvant; pulmonary resection; lung cancer

Received: 19 December 2019; Accepted: 10 March 2020; Published: 20 July 2021.

doi: 10.21037 /jovs.2020.03.07

View this article at: http://dx.doi.org/10.21037/jovs.2020.03.07

\section{Progress in immunotherapy}

The past decade has shown considerable promise in immune-checkpoint blockade as a means of augmenting antitumor immunity, placing immunotherapy alongside traditional modalities as a pillar of cancer treatment. Current checkpoint-blockade strategies are centered either on cytotoxic T lymphocyte-associated antigen-4 (CTLA-4) or programmed cell death protein-1 pathway (PD-1/PD-L1) (1).

CTLA-4 and PD-1 facilitate T-cell attenuation at different stages of the T-cell response. Immediately following T-cell receptor ligation, CTLA-4 is translocated to the T-cell surface, where it competes for binding to CD80 and CD86, ligands that could otherwise provide positive co-stimulation to the T-cell via CD-28 (2). As a result, CTLA-4 increases the activation threshold of T cells, impeding antitumor immunity by dampening the sensitivity of T-cells to self and tumor antigens (3). The PD-1 pathway, by contrast, is induced later during $\mathrm{T}$ cell activation, attenuating TCR signaling following binding by PD-L1. Cancer cells expressing PD-L1 are thereby able to evade immune detection, while tumor-infiltrating immune cells can themselves express PD-L1 in response to cytokines such as IFN- $\gamma(3)$. PD-L1 expression throughout the inflammatory tumor microenvironment can therefore lead 
to PD-1-mediated T-cell exhaustion (2).

Whereas anti-CTLA-4 monoclonal antibody therapy has earned FDA-approval for treating advanced melanoma, CTLA-4 blockade has shown minimal activity in the treatment of non-small cell lung cancer (NSCLC). Meanwhile, agents targeting the PD-1/PD-L1 axis have shown considerable efficacy in treating advanced NSCLC (4). Currently, there is significant interest in determining the therapeutic potential of combined CTLA-4 and PD-1 blockade. In CheckMate 227, a randomized, open-label, phase 3 trial by Hellman et al. first-line treatment of stage IV or recurrent NSCLC with nivolumab (anti-PD-1) plus ipilimumab (anti-CTLA-4) resulted in a longer duration of overall survival than did chemotherapy (5).

In light of the evidence for immunotherapy in treating advanced cancers, interest has risen in determining the role of immunotherapy in treating earlier stages of disease. Currently, surgical resection, often paired with neoadjuvant or adjuvant therapies, remains the most effective means of treating resectable disease. While anti-CTLA-4 and antiPD-1 were first examined as potential adjuvant therapies in resectable cancer, recent attention has turned towards the use of immunotherapy in a neoadjuvant role (6). In preclinical mouse studies, Liu and colleagues demonstrated improved long-term survival with neoadjuvant immunotherapy and surgical resection, as opposed to resection followed by adjuvant immunotherapy (7). It is thought the presence of the full tumor mass at the start of immunotherapy, as is the case in neoadjuvant immunotherapy, allows for a broader and more robust T-cell response.

\section{Immunotherapy in resectable NSCLC}

Given that PD-1 blockade has been shown to improve survival in patients with advanced lung cancer, the role of immunotherapy in resectable NSCLC has been a subject of recent interest.

In their 2018 report, Forde and colleagues describe the results of their phase II prospective trial, in which patients with resectable NSCLC received a dose of intravenous nivolumab every two weeks, with surgery scheduled four weeks after the first dose (8). In addition to evaluating the safety and feasibility of nivolumab in patients with resectable NSCLC, Forde and colleagues also examined the relationships between tumor mutational burden, tumor microenvironment, peripheral immune cell activity, and final tumor pathology.
Of the 22 patients enrolled in the study, one patient was deemed ineligible upon discovery of small cell lung carcinoma. Of 21 remaining patients, 20 received two doses of nivolumab prior to surgical resection, with one patient developing pneumonia and undergoing tumor resection after only one dose of nivolumab. Of the 20 patients to receive two doses of nivolumab, one patient underwent an incomplete tumor resection, following the discovery of tracheal invasion intraoperatively.

In their examination of safety and feasibility, Forde et al. reported adverse events in 5 of 22 patients, with no delays in planned surgery. Radiographic response was determined to be partial in $2(10 \%)$, stable disease in 18 (86\%), and disease progression in 1 (5\%). In two patients for whom the tumor appeared to increase in size, pathological evaluation of the surgical specimen demonstrated minimal or no residual tumor, suggesting that immunotherapy may be effective despite the lack of radiographic response, with immune-cell infiltration contributing to mass size.

Of the 20 patients who underwent complete surgical resection, one patient experienced an incidental death unrelated to the study, while $16(80 \%)$ were alive and recurrence-free at 12 months, and 3 (15\%) experienced disease progression. In evaluating surgical specimens from the 20 complete surgical resections, a major pathological response ( $\mathrm{mPR})$ to therapy was seen in 9 of the resected specimens (45\%), with 3 patients' specimens demonstrating a complete pathological response (pCR). A significant number of inflammatory cells were discovered in the tumor specimens, including lymphocytes, macrophages, necrotic tumor, and fibrotic tissue.

In reviewing the pathology of 15 patients who underwent pre-treatment biopsy for PD-L1, Forde et al. found major pathologic responses in both PD-L1-positive and PD-L1negative tumors. Following whole-exome sequencing of 11 patients' pre-treatment tumors, studied alongside their corresponding final pathology, higher tumor mutational burden was significantly associated with a major pathological response. An inverse relationship between tumor mean mutational burden and the percentage of residual tumor on final pathology was noted, suggesting that mutation burden is a primary determinant in the pathological response to PD-1 blockade.

To determine the effect of PD-1 blockade on T-cell activity, Forde et al. examined T-cells found both in resected tumors and peripheral blood of 9 patients, sequencing T-cell receptor- $\beta$ chain CDR3 regions (TCRseq) to determine the repertoire of $\mathrm{T}$-cell clones. In contrast to tumors that 
did not show a major pathological response, tumors that demonstrated a major pathological response were found to have a higher frequency of T-cell clones shared between the intratumoral and peripheral compartments, as well as increased clonality among T-cells. Meanwhile, in 8 of 9 patients, peripheral blood demonstrated expansion of multiple T-cell clones that were also found in the tumor, yet had not been detected in blood before treatment.

In their analysis of peripheral blood of one patient who had demonstrated a complete pathological response, T-cells within post-operative peripheral blood samples responded to a broader range of sample candidate neoantigens than did T-cells from pre-treatment blood samples. The authors hypothesized that neoadjuvant PD-1 blockade leverages the primary tumor's abundance of antigen in enabling the activation and clonal expansion of antitumor T-cells. It is thought that such T-cells can then travel systemically to various tissues, providing systemic surveillance for

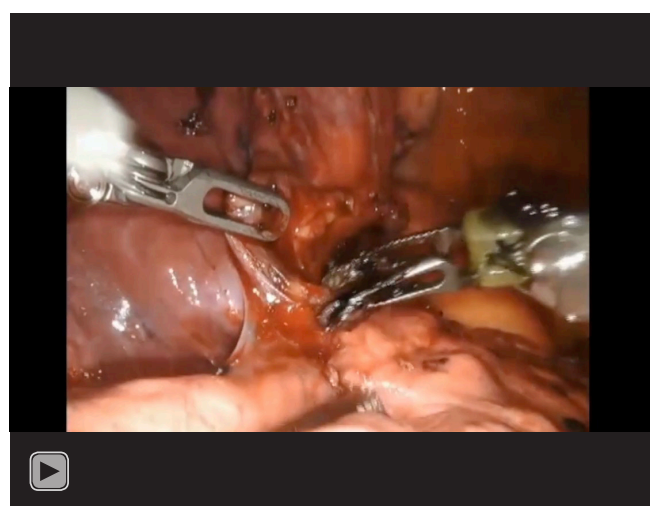

Video 1 Robotic left upper lobectomy after immunotherapy with suture reconstruction and reinforcement of the left upper lobe bronchus. micrometastases.

\section{Pulmonary resection after immunotherapy}

Induction immunotherapy poses unique technical challenges during lung resection (see Video 1). Dense fibrosis of the hilum or lymph node stations may obscure tissue planes and make structures adherent. A limited number of series have reported perioperative outcomes after immunotherapy. In general, these studies report comparable results to resection after neoadjuvant chemotherapy and/or chemoradiation. Minimally invasive approaches to resection, including the use of robotic systems, reveal a $20-54 \%$ rate of conversion to open thoracotomy. A complete resection, however, is achieved in the majority of patients and postoperative morbidity is limited in severity.

The first study that explored the use of neoadjuvant immunotherapy in the resection of lung malignancies was performed by Chaft et al. who reported a case series of five patients (Table 1) (9). The investigators address the initial concerns about the use of neo-adjuvant immunotherapy, which was centered on potential side-effects such as the development of pneumonitis and fibrosis, which could make the procedures more technically difficult. Although one of the patients had their surgery delayed by the development of pneumonitis, all five patients underwent successful tumor resection. Two patients underwent a minimallyinvasive resection, include one robotically. One of these patients converted to open thoracotomy due to "dense hilar fibrosis." The report, presented in a series of case vignettes, detail limited treatment and postoperative morbidity, without a postoperative mortality within 30-days.

The feasibility of pulmonary resection after immunotherapy as was further explored by Bott $e t a l$. who described their experience in 22 patients with primary

Table 1 Pulmonary resection after immunotherapy - perioperative safety and complications

\begin{tabular}{|c|c|c|c|c|c|c|c|c|}
\hline Authors & Year & $\begin{array}{l}\mathrm{N} \\
\text { total }^{*}\end{array}$ & $\begin{array}{l}\text { Minimally } \\
\text { invasive } \\
\text { approach }\end{array}$ & $\begin{array}{l}\text { Conversion } \\
\text { to open (\%) }\end{array}$ & $\begin{array}{l}\text { Operative } \\
\text { time (mean) }\end{array}$ & Complications & $\begin{array}{l}\text { Mortality at } \\
30 \text { days (\%) }\end{array}$ & Comment \\
\hline Bott et al. (10) & 2018 & 22 & 15 & $1(25 \%)$ & $168 \min$ & $7(32 \%)$ & $0(0 \%)$ & $\begin{array}{l}11(50 \%) \text { underwent non-anatomic } \\
\text { wedge resection }\end{array}$ \\
\hline Yang et al. (12) & 2018 & 13 & 12 & $3(23 \%)$ & NR & $9(69 \%)$ & $0(0 \%)$ & $10(77 \%)$ patients underwent lobectomy \\
\hline
\end{tabular}

*, total patients resected. R0, complete resection; min: minutes; NR, not reported. 
or metastatic lung malignancies who were treated with nivolumab, an anti-PD-1 antibody (10). Primary lung cancer was the most common diagnosis. A minimallyinvasive approach as attempted in 15 patients with only one conversion to open thoracotomy. Of note, a substantial number of patients $(11,50 \%)$ underwent a non-anatomic wedge resection. Surgical complications occurred in $32 \%$ of the patients, the most common of which was prolonged air leak. Only one patient developed pneumonitis, which occurred in the contralateral lung. Complete resection was achieved in all but one patient, and there were no mortalities at 30 and 90 days. The two-year overall and disease-free survival were $77 \%$ and $42 \%$ respectively.

This initial experience was followed by a second study in 21 patients, all with NSCLC, who were treated with two cycles of nivolumab at two and four weeks preoperatively (11). One patient had their surgery moved up after developing of post-obstructive pneumonia after their first dose of nivolumab. All other procedures occurred as scheduled, 2 weeks after the final dose. Of the 13 procedures that were planned as minimally invasive (VATS or robotic), $54 \%$ were converted to open due to dense, vascularized hilar or mediastinal adhesions-this was more common in patients with advanced disease. These initial studies demonstrated that lung resection after immunotherapy was both safe and feasible, even in patients who develop pneumonitis or mediastinal fibrosis.

In addition to reporting on surgical outcomes, the second report by Bott et al. also describes the pathological response of the tumors to nivolumab. On pre-operative CT imaging, $86 \%$ had stable disease, $5 \%$ with disease progression, and only $10 \%$ showing a partial response to the immunotherapy. However, post-operative pathology revealed that $10 \%$ of the cohort had a complete pathological response, $45 \%$ had a major pathological response (MPR), defined as $<10 \%$ viable residual tumor, and an additional $40 \%$ showing pathological down-staging.

Checkpoint inhibitors have also been studied when used in combination with neo-adjuvant chemotherapy. Yang et al. reported the results of the TOP1201 trial, a phase II trial that treated NSCLC patients with neoadjuvant paclitaxel and cisplatin/carboplatin followed by ipilimumab, an antiCTLA-4 antibody (12). The 24 patients enrolled in the study had minimal toxicity, the most common of which were pneumonitis, adrenal insufficiency, or diarrhea. Most other adverse effects seen in the group were known effects of the other chemotherapeutic agents used. Only 13 of the initial 24 patients went on to have a lung resection, however, of the 11 excluded patients, only one was excluded due to a treatment-related adverse effect. The ipilimumab group had good post-operative outcomes, with a two-year survival rate of $73 \%$, and similar morbidity and mortality to the patients treated with neo-adjuvant chemotherapy alone.

Nivolumab has also been used in combination with preoperative chemotherapy (13). The results of combination therapy are reported in the NADIM trial, an ongoing phase II clinical trial of patients with resectable stage IIIA N2-NSCLC. Current outcomes in 13 patients who have undergone surgical resection after nivolumab reveal no delays in surgery with $83 \%$ of patients having either a major or complete pathological response. Perioperative outcomes have not yet been reported.

The optimal timing of surgical intervention after neoadjuvant immunotherapy has not been well defined. In the previously mentioned trials, patients were scheduled for resection approximately 3-5 weeks after completion of induction therapy. Delays in this treatment schedule were rate, although a small number of patients had a protracted time to surgery, in part due to immunotherapy toxicities. The rationale for a relatively expedient resection is based on the premise of a transition from inflammation to fibrosis over time.

\section{Toxicity of immunotherapy}

The toxicity of immunotherapies creates new challenges for both oncologists and thoracic surgeons. Early experience of neoadjuvant immunotherapy for NSCLC provided insights into the safety profile of immune checkpoint inhibitors. In general, morbidity and mortality did not appear to increase in patients treated with neoadjuvant immunotherapy when they were compared with historical data in which induction chemotherapy was used. In a series of 20 lung resections after neoadjuvant nivolumab therapy for NSCLC, the rates of pneumonia, reintubation, prolonged air leak, and respiratory failure were lower whereas the rate of atrial fibrillation was higher than those in the Southwest Oncology Group S9900 trial, a study evaluating neoadjuvant therapy with carboplatin and paclitaxel $(11,14)$. Similarly, Bott et al. reported 22 lung resections for suspected residual pulmonary disease following immunotherapy for advancestage cancers with a morbidity profile comparable to that of lung resections for NSCLC following neoadjuvant chemoor chemoradiotherapy at the same institution $(10,15)$.

In the report of surgical outcomes of TOP1201 trial that investigated neoadjuvant ipilimumab for NSCLC, participants were compared with a historical cohort of 
NSCLC patients who underwent preoperative chemotherapy alone (TOP1201) (16). There was no trend of increased morbidity or mortality observed. An additional safety aspect of the neoadjuvant immunotherapy specific to lung resection is whether its adoption would increase technical difficulty of surgery. Dense fibrosis formed as a result of the immune response may pose significant challenge to mediastinal and hilar dissections. The operative time, a possible surrogate for technical difficulty, has not seemed to depart from reported experience with induction chemotherapy. These data offered prospects of safely employing immune checkpoint inhibitors as induction therapy, however, we anticipate the results from the ongoing large-scale trials to better characterize safety in randomized patients.

The adverse effects of CTLA-4 and PD- 1 axis inhibitors involving multiple organ-systems have been extensively described in the trials for the treatment of advanced melanoma, NSCLC, head and neck squamous cancers, and urothelial cancers (17). Some of the toxicities may adversely impact surgical outcomes or potentially delay timing to surgical interventions. Pneumonitis is estimated to have an overall incidence of $5 \%$ in patients treated with checkpoint inhibitors for melanoma or NSCLC and tends to be more common in those who received combined antiPD-1 and anti-CTLA-4 therapies (18). The incidence of endocrinopathies was found to be approximately $10 \%$ in one meta-analysis, though the ones that could lead to perioperative hemodynamic consequences such as adrenal insufficiency were rare (19). Nonetheless, these toxicities in neoadjuvant immunotherapy for NSCLC have been reported to be scarce in the currently available literature and warrant further attention as the outcomes of ongoing trials are revealed.

\section{Future trials in immunotherapy and surgery}

Given the encouraging results from the pilot studies, we are awaiting several ongoing larger trials that will further establish the safety and effectiveness profiles of neoadjuvant immunotherapy in treating resectable NSCLC. CheckMate 816 (NCT02998528) is a multicenter, phase III, randomized controlled trial that compares adding an antiPD-1 agent, nivolumab, to platinum doublet chemotherapy with chemotherapy alone (20). It was initiated in January 2017 with a current enrollment of 350 patients who have Stage IB to IIIA diseases $(20,21)$. The primary outcome measures are event-free survival (EFS) up to 69 months and pCR at the time of surgery. It is estimated to complete final data collection for primary outcome measures by April 2020. Another study investigating neoadjuvant nivolumab is the single-center (M.D. Anderson Cancer Center), phase II, NEOSTAR trial (NCT03158129) $(22,23)$. Additionally the study will explore combining nivolumab with ipilimumab, a CTLA-4 inhibitor that modulates T-cell activation via mechanisms distinct from and complementary to those of PD-1 axis inhibitors. The design was based on the result of CheckMate 012 trial, which suggested high response rate and durable response in treating advanced NSCLC using this combination (17). With an estimated enrollment of 66 patients, the trial randomizes patients into receiving neoadjuvant nivolumab, nivolumab plus ipilimumab, or nivolumab plus platinum doublet chemotherapy. The primary outcome is the mPR defined as less than or equal to $10 \%$ viable tumor cells in the resected specimen up to eight weeks. This study is expected to compete primary outcome measure data collection by July 2021 . Pembrolizumab, an anti-PD-1 antibody that has been used in combination with chemotherapy as the standard therapy in PD-L1 low advanced NSCLC, is currently being studied as a neoadjuvant and adjuvant agent in the MK-3475-671/ KEYNOTE-671 trial $(24,25)$. This phase-III study plans to recruit 786 patients with Stage II to IIIB (N2) NSCLC and will complete data collection by January 2024. The experimental arm of the study adds perioperative treatment with pembrolizumab (versus placebo in the comparator arm) to neoadjuvant chemotherapy with the primary outcome measures of EFS and overall survival up to 5 years (26).

Several other ongoing trials are examining anti-PD-L1 antibodies such as atezolizumab and durvalumab. The phase-III IMpower030 trial that involves atezolizumba recruits from the same patient population as the MK3475-671/KEYNOTE-671 trial with an enrollment goal of 374 patients. The neoadjuvant therapies in the study arms consist of platinum doublet chemotherapy and either atezolizumab or placembo comparator. The primary outcome of the study measures $\mathrm{mPR}$ at the time of surgery, as well as investigator-assessed EFS at approximately seven years, projecting the completion of data collection in 2025. A similar study using durvalumab is being conducted in the trial identified as NCT03800134, where only mPR at approximately 15 weeks after first dose will be examined as the primary outcome measure (27).

\section{Acknowledgments}

Funding: None. 


\section{Footnote}

Provenance and Peer Review: This article was commissioned by the Guest Editor (Jean-Marc Baste) for the series "Robotic Assisted Thoracic Surgery: Adavanced Procedures in Lung and Mediastinum: From Postinduction TTT (immunotherapy) to Sleeve Resection, Complex Segmentectomies and Extended Thymectomy for Myasthenia Gravis" published in Journal of Visualized Surgery. The article has undergone external peer review.

Conflicts of Interest: All authors have completed the ICMJE uniform disclosure form (available at https://jovs. amegroups.com/article/view/10.21037/jovs.2020.03.07/ coif). The series "Robotic Assisted Thoracic Surgery: Adavanced Procedures in Lung and Mediastinum: From Post-induction TTT (immunotherapy) to Sleeve Resection, Complex Segmentectomies and Extended Thymectomy for Myasthenia Gravis" was commissioned by the editorial office without any funding or sponsorship. RJC serves as an unpaid editorial board member of fournal of Visualized Surgery from February 2018 to January 2022. The authors have no other conflicts of interest to declare.

Ethical Statement: The authors are accountable for all aspects of the work in ensuring that questions related to the accuracy or integrity of any part of the work are appropriately investigated and resolved. All procedures performed in this study were in accordance with the Helsinki Declaration (as revised in 2013). The manuscript is waived from patient informed consent according to the ethics committee or institutional review board.

Open Access Statement: This is an Open Access article distributed in accordance with the Creative Commons Attribution-NonCommercial-NoDerivs 4.0 International License (CC BY-NC-ND 4.0), which permits the noncommercial replication and distribution of the article with the strict proviso that no changes or edits are made and the original work is properly cited (including links to both the formal publication through the relevant DOI and the license). See: https://creativecommons.org/licenses/by-nc-nd/4.0/.

\section{References}

1. Wei SC, Levine JH, Cogdill AP, et al. Distinct Cellular Mechanisms Underlie Anti-CTLA-4 and Anti-PD-1 Checkpoint Blockade. Cell 2017;170:1120-33.e17.
2. Ribas A, Wolchok JD. Cancer immunotherapy using checkpoint blockade. Science 2018;359:1350-5.

3. Seidel JA, Otsuka A, Kabashima K. Anti-PD-1 and AntiCTLA-4 Therapies in Cancer: Mechanisms of Action, Efficacy, and Limitations. Front Oncol 2018;8:86.

4. Herzberg B, Campo MJ, Gainor JF. Immune Checkpoint Inhibitors in Non-Small Cell Lung Cancer. The Oncologist 2017;22:81-8.

5. Hellmann MD, Rizvi NA, Goldman JW, et al. Nivolumab plus ipilimumab as first-line treatment for advanced nonsmall-cell lung cancer (CheckMate 012): results of an open-label, phase 1, multicohort study. Lancet Oncol 2017;18:31-41.

6. O'Donnell JS, Hoefsmit EP, Smyth MJ, et al. The Promise of Neoadjuvant Immunotherapy and Surgery for Cancer Treatment. Clin Cancer Res 2019;25:5743-51.

7. Liu J, Blake SJ, Yong MCR, et al. Improved Efficacy of Neoadjuvant Compared to Adjuvant Immunotherapy to Eradicate Metastatic Disease. Cancer Discov 2016;6:1382-99.

8. Forde PM, Chaft JE, Smith KN, et al. Neoadjuvant PD-1 Blockade in Resectable Lung Cancer. N Engl J Med 2018;378:1976-86.

9. Chaft JE, Hellmann MD, Velez MJ, et al. Initial Experience With Lung Cancer Resection After Treatment With T-Cell Checkpoint Inhibitors. Ann Thorac Surg 2017;104:e217-8.

10. Bott MJ, Cools-Lartigue J, Tan KS, et al. Safety and Feasibility of Lung Resection After Immunotherapy for Metastatic or Unresectable Tumors. Ann Thorac Surg 2018;106:178-83.

11. Bott MJ, Yang SC, Park BJ, et al. Initial results of pulmonary resection after neoadjuvant nivolumab in patients with resectable non-small cell lung cancer. J Thorac Cardiovasc Surg 2019;158:269-76.

12. Yang CJ, McSherry F, Mayne NR, et al. Surgical Outcomes After Neoadjuvant Chemotherapy and Ipilimumab for Non-Small Cell Lung Cancer. Ann Thorac Surg 2018;105:924-9.

13. Provencio M, Nadal E, Insa A, et al. Neoadjuvant chemoimmunotherapy for the treatment of stage IIIA resectable non-small-cell lung cancer (NSCLC): A phase II multicenter exploratory study — Final data of patients who underwent surgical assessment. J Clin Oncol 2019;37:8509.

14. Pisters KMW, Vallières E, Crowley JJ, et al. Surgery with or without preoperative paclitaxel and carboplatin in earlystage non-small-cell lung cancer: Southwest Oncology Group Trial S9900, an intergroup, randomized, phase III 
trial. J Clin Oncol 2010;28:1843-9.

15. Barnett SA, Rusch VW, Zheng J, et al. Contemporary results of surgical resection of non-small cell lung cancer after induction therapy: A review of 549 consecutive cases. J Thorac Oncol 2011;6:1530-6.

16. Fang L, Wang L, Wang Y, et al. Video assisted thoracic surgery vs. thoracotomy for locally advanced lung squamous cell carcinoma after neoadjuvant chemotherapy. J Cardiothorac Surg 2018;13:128.

17. Champiat S, Lambotte O, Barreau E, et al. Management of immune checkpoint blockade dysimmune toxicities: a collaborative position paper. Ann Oncol 2016;27:559-74.

18. Naidoo J, Wang X, Woo KM, et al. Pneumonitis in Patients Treated With Anti-Programmed Death-1/ Programmed Death Ligand 1 Therapy. J Clin Oncol 2017;35:709-17.

19. Barroso-Sousa R, Barry WT, Garrido-Castro AC, et al. Incidence of endocrine dysfunction following the use of different immune checkpoint inhibitor regimens a systematic review and meta-analysis. JAMA Oncol 2018;4:173-82.

20. A Neoadjuvant Study of Nivolumab Plus Ipilimumab or Nivolumab Plus Chemotherapy Versus Chemotherapy Alone in Early Stage Non-Small Cell Lung Cancer (NSCLC) - Tabular View - ClinicalTrials.gov. Available online: https://clinicaltrials.gov/ct2/show/record/ NCT02998528?view=record

21. Felip E, Brahmer J, Broderick S, et al. P2.16-03 CheckMate 816: A Phase 3 Trial of Neoadjuvant Nivolumab Plus Ipilimumab or Chemotherapy vs Chemotherapy in Early-Stage NSCLC.J Thorac Oncol 2018;13:S831-2.

22. Cascone T, William WN, Weissferdt A, et al. Neoadjuvant nivolumab $(\mathrm{N})$ or nivolumab plus ipilimumab (NI) for resectable non-small cell lung cancer (NSCLC): Clinical and correlative results from the NEOSTAR study. Available online: https://ascopubs.org/doi/abs/10.1200/ JCO.2019.37.15_suppl.8504

23. Nivolumab With or Without Ipilimumab or Chemotherapy in Treating Patients With Previously Untreated Stage I-IIIA Non-small Cell Lung Cancer - Tabular View - ClinicalTrials.gov. Available Available online: https://clinicaltrials.gov/ct2/show/record/ NCT03158129

24. Gandhi L, Rodríguez-Abreu D, Gadgeel S, et al. Pembrolizumab plus Chemotherapy in Metastatic NonSmall-Cell Lung Cancer. N Engl J Med 2018;378:2078-92.

25. Efficacy and Safety of Pembrolizumab (MK-3475) With Platinum Doublet Chemotherapy as Neoadjuvant/ Adjuvant Therapy for Participants With Resectable Stage II, IIIA, and Resectable IIIB (T3-4N2) Non-small Cell Lung Cancer (MK-3475-671/KEYNOTE-671) Tabular View - ClinicalTrials.gov. Available online: https:// clinicaltrials.gov/ct2/show/record/NCT03425643

26. Fernando HC, Yang J, Ferraro GL, et al. Randomized, double-blind phase 3 study evaluating neoadjuvant platinum-based chemotherapy with perioperative pembrolizumab or placebo in resectable stage IIB or IIIA NSCLC: KEYNOTE-671. J Clin Oncol 2018;36:TPS8583.

27. A Study of Neoadjuvant/Adjuvant Durvalumab for the Treatment of Patients With Resectable Non-small Cell Lung Cancer - Tabular View - ClinicalTrials.gov. Available online: https://clinicaltrials.gov/ct2/show/record/ NCT03800134 doi: 10.21037/jovs.2020.03.07

Cite this article as: Sasankan P, Hyde J, Wang S, Geraci TC, Cerfolio RJ. Pulmonary resection after immunotherapy. J Vis Surg 2021;7:29. 